\title{
Antioxidant Activity of the Phenolic Leaf Extracts from Monechma ciliatum in Stabilization of Corn Oil
}

\begin{abstract}
The total phenolic content and the antioxidan potential of methanolic extract (ME), ethyl acetate extract (EAE), and hexane extract (HE) from Monechma ciliatum leaves (MCL) were evaluated. The Folin-Ciocalteu, b-carotene bleaching, the 2,2-diphenyl-1-picrylhydrazyl (DPPH) radical scavenging and the accelerated oxidation methods were used for evaluation. Both the extraction yield and the antioxidant activity (AOA) were strongly dependent on the solvent. Among the extracts, ME exhibited highest total phenolic compounds (TPC) and IC50 values for DPPH, followed by EAE and HE, respectively. Peroxide value (PV), anisidine value (AV) conjugated dienes (CD), and thiobarbituric acid reactive substances (TBARS) were taken as the parameters for evaluation of stabilization efficacy of MCL extracts and results revealed MCL to be a potent antioxidant for the stabilization of corn oil. As a general trend, increased AOA was observed for increased extract concentration. The predominant phenolic compounds identified by HPLC-DAD in MCL extracts were p-coumaric acid, vanillin and ferulic acid.
\end{abstract}

Keyword: Antioxidant activity; $\beta$-Carotene bleaching assay; 2,2-Diphenyl-1-picrylhydrazyl (DPPH); Monechma ciliatum; Oil oxidation; Total phenolic content 